\title{
Guides de bonnes pratiques d'hygiène dans le secteur des oléagineux
}

\author{
Sylvie DAUGUET ${ }^{1}$ \\ Morgane SAILLARD ${ }^{2}$ \\ Katell CRÉPON ${ }^{3}$ \\ ${ }^{1}$ CETIOM, \\ 11 rue Monge, \\ Parc industriel, \\ 33600 Pessac, \\ France \\ $<$ dauguet@cetiom.fr> \\ 2 FNCG, \\ 66 rue de la Boétie \\ 75008 Paris, \\ France \\ $<$ m.saillard@fncg.fr $>$ \\ ${ }^{3}$ Coop de France Métiers du Grain, \\ 43 Rue Sedaine \\ CS 91115 \\ 75538 Paris Cedex 11, \\ France
}

Article reçu le 4 septembre 2012

Accepté le 10 septembre 2012

Applicable depuis 2006, le Paquet Hygiène est une réglementation européenne qui prend en compte "de la fourche à la fourchette "I'hygiène des denrées alimentaires et aliments pour animaux afin de protéger la santé du consommateur. Elle est mise en œuvre dans tous les pays membres de I'UE et doit être respectée pour les importations en provenance de pays tiers. L'idée centrale du Paquet Hygiène est de renforcer la responsabilité de chaque

\begin{abstract}
Guides to good practice for hygiene within the French oilseed food chain

According to the Hygiene Package Regulations, European Member States encourage the development and dissemination of national guides to good practice for hygiene and for the application of HACCP principles. These guides are developed by food business sectors. Within the oilseed crops food chain, such guides are developed at different stages: primary production of oilseed crops, collection and storage of seeds, oilseeds crushing and refining industry. This article presents these three guides developed in France by the federations of concerned operators. We describe how these guides are constructed, their scope, their progress in the validation process by the authorities, the health hazards identified, best practices by sector and HACCP study.
\end{abstract}

Key words: guide to good practice, hygiene, $H A C C P$, oilseed crop, grain storage, vegetable oil industry

acteur de la filière concernant la sécurité sanitaire des produits qu'il met sur le marché. Cette réglementation encourage la rédaction, de façon collective, de guides de bonnes pratiques d'hygiène (GBPH). Un guide de bonnes pratiques $d^{\prime}$ 'hygiène et d'application des principes HACCP est un document de référence, d'application volontaire, conçu par une branche professionnelle pour les professionnels de son secteur selon les recommandations de I'Avis aux professionnels de I'alimentation relatif aux guides de bonnes pratiques d'hygiène et d'application des principes HACCP (JO $\left.n^{\circ} 138\right)$ (Anon, 2005b). II rassemble les recommandations qui, aux étapes de la chaîne alimentaire et pour les denrées alimentaires ou aliments pour animaux qu'il concerne, doivent aider au respect des règles d'hygiène fixées par les règlements du Paquet Hygiène (règlement (CE) n 852/2004 (Anon, 2004), règlement (CE) $n^{\circ} 183 / 2005$ (Anon, 2005a)), et aider à l'application des principes HACCP (Hazard Analysis Critical Control Point ou Analyse de dangers et maîtrise des points critiques $^{1}$ ). Le Paquet Hygiène prévoit que les procédures selon les principes de I'HACCP soient suivies par tous les opérateurs de la chaîne alimentaire, à l'exception de la production primaire (production agricole), en raison de la diversité des activités possibles au sein des exploitations agricoles et de leur taille. Ces guides doivent être validés par les pouvoirs publics au niveau national ou européen, après expertise-évaluation par l'Agence de sécurité sanitaire des aliments (ANSES) au niveau français, et par le CPCASA (Comité permanent de la

\footnotetext{
${ }^{1}$ L'HACCP est un système qui identifie, évalue et maîtrise les dangers significatifs au regard de la sécurité des aliments. II peut s'agir de dangers biologiques (mycotoxines, micro-organismes. . .), chimiques (résidus de produits phytopharmaceutiques. . .) ou physiques (corps étrangers. . .). Le concept HACCP a été mis au point au départ par la NASA pour garantir la sécurité microbiologique des aliments des astronautes. Le système actuel repose sur des documents élaborés dans le cadre du Codex Alimentarius. II repose sur sept étapes et douze principes. Le système HACCP est également à la base du système normatif de management de la sécurité des aliments (NF EN ISO 22000).
}

Pour citer cet article : Dauguet S, Saillard M, Crépon K. Guides de bonnes pratiques d'hygiène dans le secteur des oléagineux. OCL 2013 ; 20(2) : 124-130. doi : 10.1684/ocl.2012.0477 
chaîne alimentaire et de la santé animale) au niveau européen.

Les opérateurs de la filière oléagineuse sont notamment concernés par trois $\mathrm{GBPH}$, à différents stades: le premier concernant la production de grandes cultures s'adresse aux agriculteurs (en cours d'élaboration), le deuxième concernant les opérations de collecte et stockage des grains s'adresse aux organismes stockeurs (validé), et le troisième concernant la transformation des graines oléagineuses s'adresse aux industries de la trituration et du raffinage (en cours de validation).

\section{Guide de bonnes pratiques d'hygiène pour la production de grandes cultures}

Pour éviter à chaque agriculteur d'identifier individuellement les risques sanitaires sur son exploitation et les mesures de maîtrise nécessaires, les filières françaises de grandes cultures ont décidé d'élaborer collectivement un guide de bonnes pratiques d'hygiène pour la production de grandes cultures. Ce guide est en cours d'élaboration : une synthèse sur l'analyse des dangers et des extraits du futur guide ont été remis à la DGCCRF en septembre 2011, et sont en cours d'expertise par l'ANSES. Lorsque le retour de cette expertise sera fait auprès des rédacteurs, des modifications nécessaires pourront être apportées pour permettre ensuite la finalisation du guide.

La rédaction de ce guide pour les grandes cultures a été assurée par un groupe de travail composé de plusieurs organismes : des instituts techniques et interprofessions des filières concernées (Arvalis chargé de la coordination, CETIOM, UNIP, ITB, ITAB, ONIDOL, CNIPT, GIPT, IRTAC), des représentants des producteurs (AGPB, AGPM, FOP, CGB, UNPT), ainsi que I'APCA, FranceAgriMer, INRA, Coop de France, FNA, FNEDT, FEDEPOM, et en collaboration avec I'administration (DGAL, DGCCRF, DGS).

\section{Construction du guide}

Dans le cas des guides relatifs à la production agricole, la démarche est quelque peu différente des autres guides concernant les industries agro-alimentaires, puisqu'il a été accepté et démontré (projet CASDAR 2007-2010) que, dans le cas de la production agricole, I'ensemble des principes de I'HACCP ne sont pas applicables (Cerf, 2011). Aucun point critique pour la maîtrise des dangers (CCP) au sens strict $n$ 'est mis en évidence dans le cas de la production agricole et ainsi, la mise en place de bonnes pratiques d'hygiène (dénommées aussi programmes prérequis ou PRP) suffirait à créer un environnement hygiénique qui ne nécessite pas une maîtrise supplémentaire des dangers.

Le GBPH pour la production de grandes cultures a été rédigé en tenant compte des trois principes suivants:

- lister les contaminants pouvant se retrouver dans la denrée alimentaire ou I'aliment pour animaux et qui ont un impact sur la santé du consommateur ; - déterminer les bonnes pratiques d'hygiène permettant de réduire à un niveau acceptable la présence des contaminants définis précédemment ; - lister les enregistrements et/ou documents permettant de prouver la mise en place de bonnes pratiques.

Il s'appuie sur une première étape d'analyse des dangers pour l'ensemble des productions, qui a fait l'objet d'un document séparé soumis à validation par les pouvoirs publics et à expertise par I'ANSES (document qui n'est pas destiné à être publié). À partir de la liste des contaminants retenus pour chaque culture, ou groupe de cultures, la deuxième étape a consisté à identifier les pratiques de maîtrise et les enregistrements correspondants, regroupés dans le GBPH proprement dit qui fera l'objet d'une publication après validation officielle.

\section{Champ d'application}

Ce guide couvre les productions de grandes cultures qui sont destinées à I'alimentation humaine et à l'alimentation des animaux qui entrent dans la chaîne alimentaire, hors fourrages et autoconsommation : graines oléagineuses (colza, tournesol, soja, lin), céréales à paille, maïs, sorghos, protéagineux, légumes secs, betteraves industrielles et pommes de terre. II couvre aussi tous les modes de production, y compris l'agriculture biologique. Ce GBPH concerne tous les opérateurs intervenant dans le processus de productions primaires (agriculteurs, entrepreneurs de travaux agricoles, CUMA, prestataires de services associés...). II s'applique de l'implanta- tion de la culture à l'expédition hors de l'exploitation, lorsque celle-ci est sous la responsabilité de l'agriculteur et il couvre également le séchage, le stockage, le transport, la manutention et le conditionnement sur l'exploitation (figure 1).

\section{Les dangers retenus et bonnes pratiques d'hygiene}

La liste des dangers retenus dans le GBPH de production des grandes cultures est présentée dans le figure 2, mais à noter qu'elle est susceptible d'être modifiée.

Les bonnes pratiques d'hygiène qui seront recommandées dans le futur GBPH pour la production de grandes cultures seront tout d'abord générales, pour créer un environnement hygiénique dans l'exploitation, sur la gestion des intrants, des déchets, les modes de stockage et de transport. . Elles concerneront également des pratiques agronomiques permettant de réduire certains dangers tels que la production de mycotoxines au stade culture, ou des pratiques lors du stockage à la ferme permettant de conserver les graines dans de bonnes conditions sans moisissures ni risques de contaminations par des résidus d'insecticides post-récolte.

Depuis l'entrée en vigueur du Paquet Hygiène, des éléments relatifs à I'hygiène des productions végétales sont également contrôlés dans le cadre de la conditionnalité des aides PAC, dans le domaine "Santé-Productions végétales ". II s'agit notamment de l'utilisation des produits phytopharmaceutiques : contrôle du respect des conditions d'emploi, tenue d'un registre phytosanitaire, existence d'un local ou d'une armoire de stockage des produits phytopharmaceutiques, respect des limites maximales de résidus de pesticides dans les denrées végétales produites. II est également vérifié qu'est enregistrée toute apparition d'organismes nuisibles ou maladies susceptibles d'affecter la sûreté des productions végétales et ayant une incidence sur la santé humaine (fusarioses, aspergillus, ergot du seigle), et que sont conservés les résultats de toute analyse d'échantillons de végétaux revêtant une importance pour la santé humaine. En cas d'utilisation de semences OGM pour I'alimentation des animaux, celles-ci doivent également être enregistrées. 


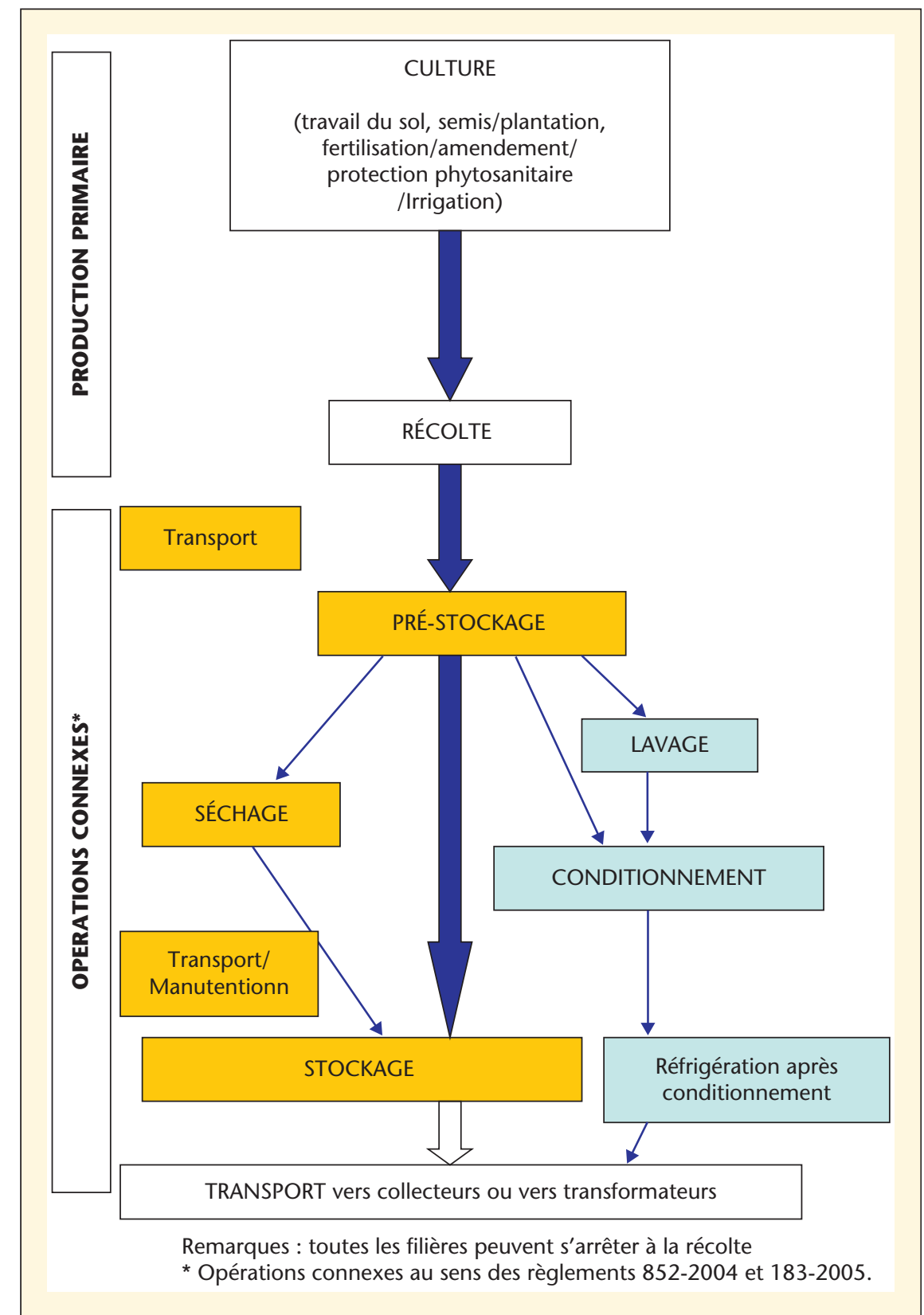

Figure 1. Schéma des opérations sous la responsabilité de l'agriculteur prises en compte dans le $G B P H$ (Guide de bonnes pratiques d'hygiene) grandes cultures (opérations connexes optionnelles selon cultures).

\section{Conclusion}

Ce GBPH grandes cultures, lorsqu'il sera publié, proposera donc des bonnes pratiques réalistes et applicables par I'ensemble des producteurs concernés. D'application volontaire, il permettra aux agriculteurs de ne pas avoir à élaborer individuellement leur propre $\mathrm{GBPH}$. II servira également de référence tant pour l'appui technique que pour les contrôles. pratiques d'hygiène et d'application du système HACCP (GBPH) depuis 2004, date de validation de la première édition du guide. Ce guide avait alors été rédigé dans le cadre réglementaire de la directive 93/43/CEE, relative à l'hygiène des denrées alimentaires. Bien que le champ d'application de cette directive soit limité aux denrées alimentaires, ce premier GBPH pour la collecte et le stockage des grains intégrait déjà des notions réglementaires spécifiques des aliments pour animaux. Néanmoins, l'entrée en vigueur des textes du Paquet Hygiène nécessitait de revoir cette version (tableau 1).

La révision du guide a été menée à partir de 2007 par une équipe pluridisciplinaire rassemblant différentes fonctions présentes chez les organismes stockeurs (représentants de la direction, responsables commercialisation, responsables qualité, responsables collecte, responsables exploitation), des experts des instituts techniques, des interprofessions ou des offices publics (ARVALIS - Institut du Végétal, CETIOM, ONIDOL, UNIP, FranceAgriMer) et des représentants du secteur de la collecte, du stockage et de la commercialisation du grain (COOP de FRANCE - Métiers du grain, FNA, SYNACOMEX) chargés de l'animation et de la coordination du travail.

Une première version du guide révisée a été adressée à la DGCCRF (administration pilote) en décembre 2008. L'AFSSA (par la suite ANSES) a été saisie en août 2009 et a rendu un avis sur l'analyse des dangers et les mesures de maîtrise proposées en mars 2010. Les remarques formulées par l'ANSES et par l'administration ayant été prises en compte, le guide a été présenté au CNC en décembre 2011 et a reçu un avis de validation par l'administration en mai 2012 (JORF du 8 mai 2012). II est publié à La Documentation française sous le titre "Guide de bonnes pratiques d'hygiène pour la collecte, le stockage, la commercialisation et le transport de céréales, d'oléagineux et de protéagineux " et est signé par COOP de FRANCE, FNA et SYNACOMEX.

\section{Construction du guide}

La structure du GBPH est conforme à la norme NF V01-001 concernant la "méthodologie pour l'élaboration des guides de bonnes pratiques d'hygiene et d'application des principes HACCP ». Le 


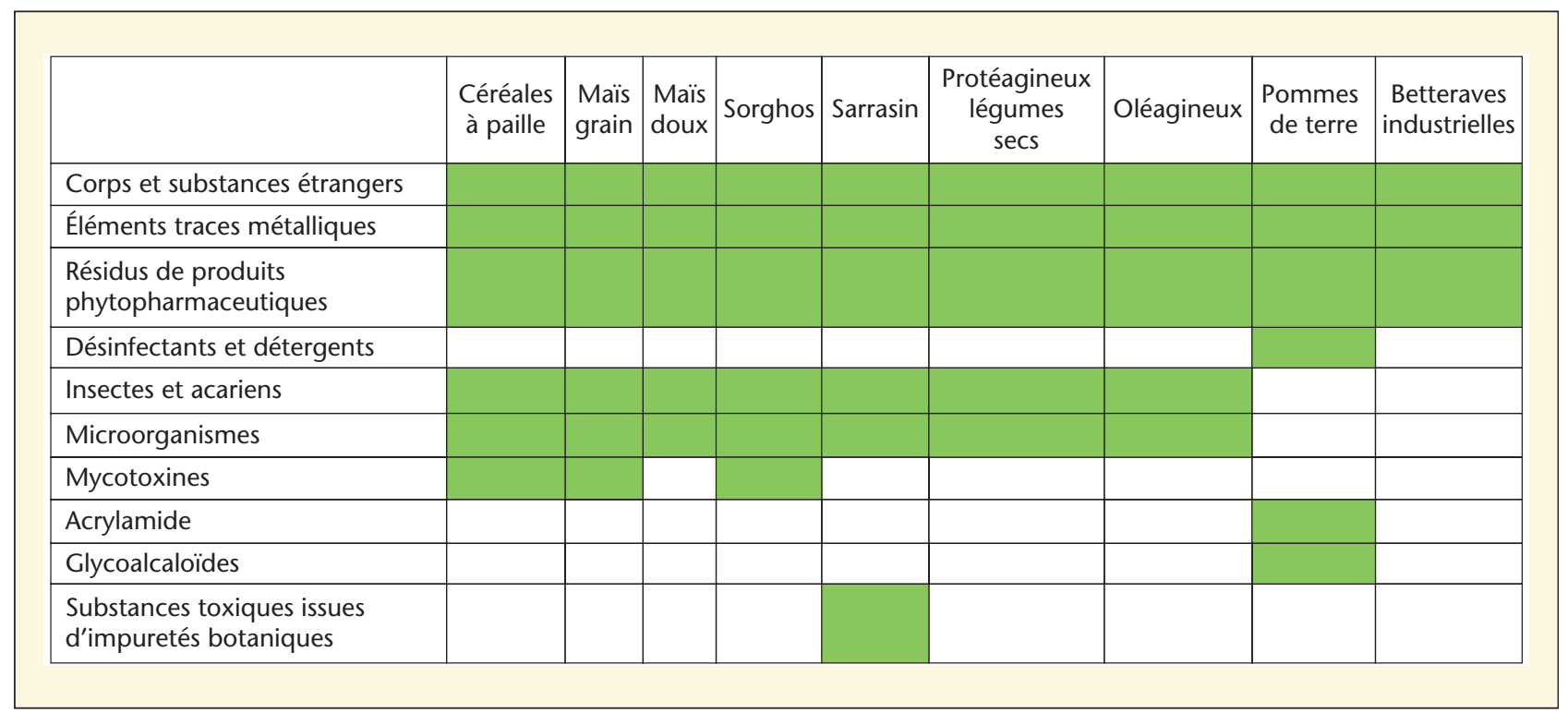

Figure 2. Liste de dangers pris en compte pour les grandes cultures (susceptible de modifications, en cours de validation).

guide est donc scindé en deux parties, une première relative aux bonnes pratiques d'hygiène dont la mise en place précède I'application du système $\mathrm{HACCP}$, et une deuxième proposant une application des principes HACCP au secteur de la collecte et du stockage. Ces deux parties renvoient à 10 annexes concernant l'étude HACCP (annexes 1 à 6), la terminologie (annexe 7), la réglementation et la bibliographie (annexe 8) ou spécifiant certains points techniques comme le transport des marchandises (annexe 9) ou la qualité de l'eau (annexe 10).

\section{Champ d'application}

Le guide couvre l'ensemble des produits habituellement collectés et stockés par

Tableau 1. Comparaison entre les deux versions du GBPH pour la collecte et le stockage des grains.

\begin{tabular}{|ll|}
\hline GBPH, version 1 (2004) & GBPH, version 2 (2012) \\
\hline Cadre réglementaire & \\
\hline $\begin{array}{l}\text { Directive 93/43/CEE (hygiène } \\
\text { des denrées alimentaires) }\end{array}$ & $\begin{array}{l}\text { Règlements 178/2002/CE, 852/2004 } \\
\text { (hygiène des denrées alimentaires), } \\
183 / 2005 \text { (hygiène des aliments pour animaux) }\end{array}$ \\
\hline Champ application & Céréales et issues de céréales non transformées \\
\hline Céréales & Protéagineux \\
\hline Protéagineux & Oléagineux \\
\hline Oléagineux & Biologiques : insectes et acariens, rongeurs, \\
\hline Dangers considérés & $\begin{array}{l}\text { volatiles et leurs traces macroscopiques, } \\
\text { moisissures, ergot du seigle, mycotoxines, } \\
\text { salmonelles, Bacillus cereus sensu lato, Bacillus } \\
\text { subtilis et Bacillus licheniformis }\end{array}$ \\
\hline $\begin{array}{l}\text { Biologiques : insectes et acariens, } \\
\text { macroscopiques, moisissures, } \\
\text { mycotoxines, salmonelles, } \\
\text { Bacillus cereus }\end{array}$ & $\begin{array}{l}\text { Chimiques : métaux lourds, résidus d'insecticides } \\
\text { de stockage, résidus de détergents et produits } \\
\text { de désinfection, dioxines, HAP et PCB }\end{array}$ \\
\hline Chimiques : métaux lourds, \\
résidus d'insecticides, dioxines
\end{tabular}

\section{Les bonnes pratiques d'hygiene}

L'ensemble des bonnes pratiques d'hygiène recommandées pour les opérateurs de collecte et de stockage est rassemblé dans la partie 1 du guide et structuré en 5 chapitres complémentaires permettant d'identifier les activités couvertes par l'opérateur luimême (compte-propre) ou par un soustraitant :

- Les bonnes pratiques générales $d^{\prime}$ hygiène ( $s^{\prime}$ appliquant à tous les opérateurs) regroupent tous les points relatifs aux locaux, aux plans de surveillance, à la formation et à l'hygiène du personnel ;

- Les bonnes pratiques (BP) relevant de I'activité commerciale listent les obligations et recommandations en termes d'enregistrement des opérateurs, de traçabilité et d'étiquetage des marchandises, de formation du personnel 


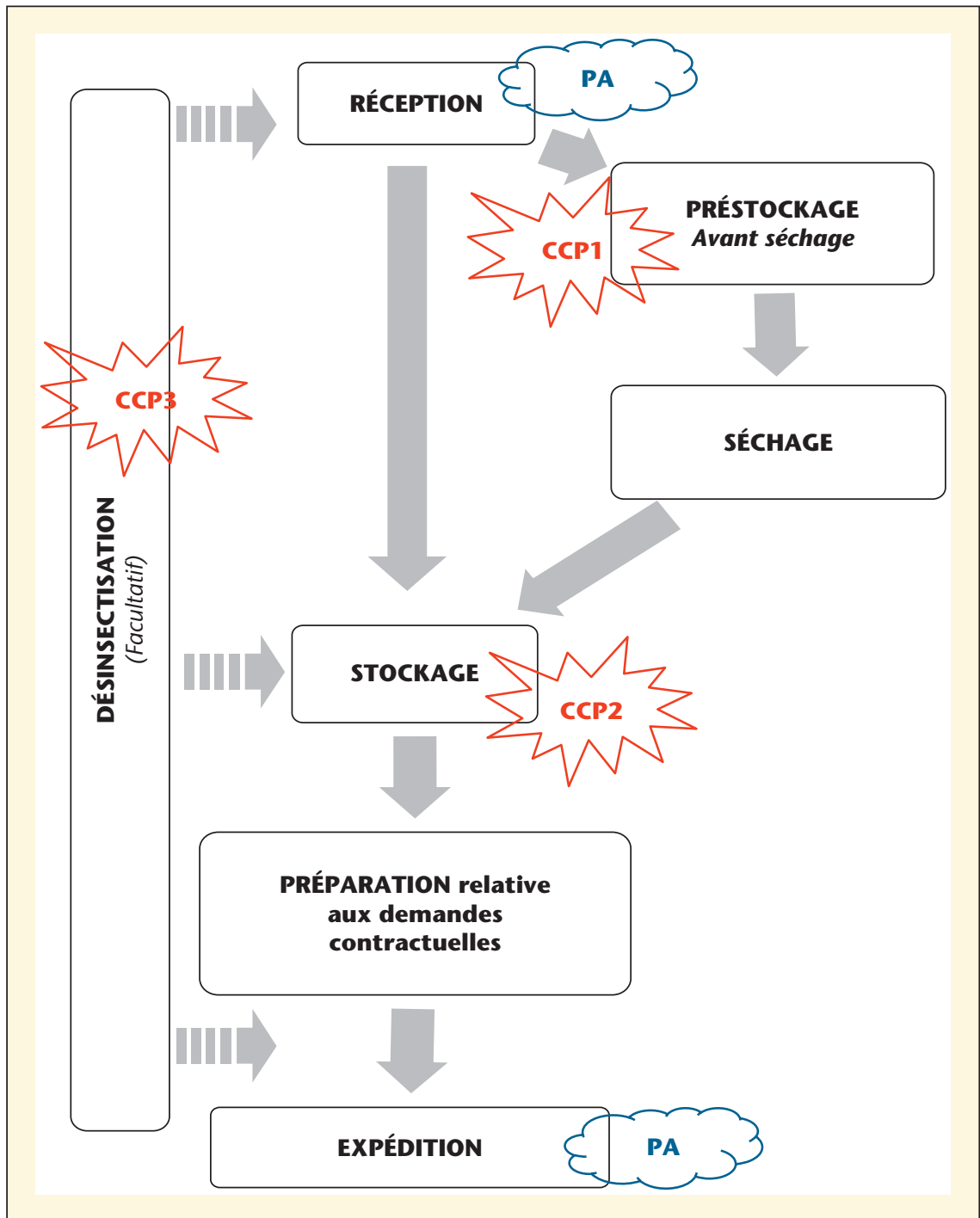

Figure 3. Diagramme des étapes, points d'attention (PA) et CCP (points critiques pour la maitrise des dangers) pour la collecte et le stockage des grains.

commercial et/ou du personnel chargé de l'expédition...;

- Les BP relatives aux opérations de collecte et réception des grains traitent de l'entretien des abords des silos et fosse de réception, des relations avec les livreurs, des contrôles à réception ;

- Les BP de stockage concernent la conception et l'entretien des installations de stockage, les opérations de ventilation et transilage des grains, la surveillance du stockage, la protection des grains contre les nuisibles (insectes, rongeurs, volatiles) ;

- Les BP relatives aux opérations d'expédition et de transport traitent des vérifications et contrôles lors de l'expédition, du nettoyage des conte-
- Chimique : métaux lourds, résidus d'insecticides de stockage, résidus de détergents et produits de désinfection, dioxines, HAP et PCB ;

- Physique : corps étrangers, radioactivité ;

- Allergène.

Chaque danger dispose d'une fiche descriptive sur la nature, l'origine, les caractéristiques, les conditions d'apparition et de développement du danger et la réglementation qui s'applique, le cas échéant. Tous les dangers ont fait I'objet d'une évaluation en considérant leur probabilité d'apparition (F), leur facilité de détection (D) et leur gravité (G) avec une échelle allant de 1 à 4 ceci afin de déterminer les dangers significatifs. Ont été retenus les dangers dont la gravité est critique, c'est-à-dire pouvant affecter la santé du consommateur, ou dont le risque $\left(F^{*} D^{*} G\right)$ est supérieur à 12. Pour chaque danger des mesures de maîtrise sont recommandées. Notons que la maîtrise du danger "mycotoxines de champ" nécessite de combiner plusieurs mesures de maîtrise qui débutent avant même la réception de la culture, puis aux étapes réception, préstockage, stockage et expédition. Ces moyens de maîtrise sont rassemblés dans un tableau " point d'attention - mycotoxines de champ ". Enfin, trois CCP (points critiques pour la maîtrise des dangers) ont été déterminés (figure 3 ) : l'étape préstockage pour la maîtrise des mycotoxines (CCP 1), l'étape stockage pour la maîtrise des mycotoxines de stockage (CCP 2) et l'étape désinsectisation pour la maîtrise des résidus $d$ 'insecticides de stockage (CCP 3).

Le plan HACCP détaille pour chaque CCP les mesures préventives, les limites critiques, la surveillance du CCP et les mesures correctives. Les graines oléagineuses sont particulièrement concernées par le troisième $C C P$, car il peut y avoir contamination des graines par une fuite du matériel de désinsectisation ou un défaut dans la pratique de désinsectisation. En effet, ces traitements insecticides peuvent être pratiqués sur céréales dans les silos de stockage; par contre, ils ne sont pas autorisés sur graines oléagineuses, $\mathrm{d}$ 'où des limites maximales de résidus très basses qui peuvent être dépassées en cas de contamination croisée accidentelle. 


\section{Conclusion}

Les organismes stockeurs, coopératives ou négociants, disposent désormais d'un guide de bonnes pratiques $d^{\prime}$ hygiène validé conforme aux dispositions règlementaires du Paquet Hygiène. Ce guide, d'application volontaire, est un outil dont la vocation est d'aider les entreprises à appliquer la règlementation et à maîtriser les risques sanitaires propres à ce secteur. Pour faciliter I'application des bonnes pratiques d'hygiène dans les entreprises, COOP de FRANCE a rédigé un guide d'application au silo, destiné aux personnels de silo et aux saisonniers. Ce guide, complémentaire du GBPH, recense de manière plus simple les bonnes pratiques d'hygiène à mettre en œuvre à chaque étape. Enfin, sur la base du GBPH, COOP de FRANCE, la FNA et le SYNACOMEX ont développé un référentiel de certification, baptisé CSAGTP (Charte Sécurité Alimentaire/Good Trading Practice), auditable et certifiable, pour permettre aux entreprises de faire valider, par un auditeur indépendant, la mise en œuvre dans leur entreprise des bonnes pratiques d'hygiène. Ce référentiel, et la liste des entreprises certifiées, est consultable sur le site du Syndicat de Paris (http://charte.incograin.com/fr/default.asp). Tous ces outils concourent à une meilleure maîtrise de la qualité sanitaire des céréales et des graines oléagineuses et protéagineuses, bénéfique à l'ensemble de la filière.

\section{Guide de bonnes pratiques d'hygiène et d'application des principes HACCP de la trituration et raffinage des graines et fruits oléagineux}

Le Guide de Bonnes Pratiques d'Hygiène et d'application des principes HACCP a été rédigé par Huileries de France et ses adhérents, avec le concours des instituts techniques CETIOM et ITERG, et de I'interprofession ONIDOL. Huileries de France regroupe les fabricants d'huiles et de tourteaux, qui possèdent une dizaine de sites de trituration et autant de raffinage répartis sur toute la France. Suivant les remarques de I'ANSES sur une première version, Huileries de France a complété le guide et a notamment actualisé l'analyse de risque au vu des contaminants nouvellement réglementés. La dernière version du guide a été envoyée à la DGCCRF en juin 2012, pour être évalué par l'ANSES.

\section{Construction du guide}

Le guide explicite en introduction le secteur de l'huilerie, avec des schémas de trituration et de raffinage et la liste des matières premières mises en œuvre. Un premier chapitre donne les recommandations en matière des bonnes pratiques $\mathrm{d}$ 'hygiène pour les industriels réalisant les opérations de trituration et/ou raffinage. Le deuxième chapitre applique les principes du système HACCP à partir des dangers potentiels retenus pour le secteur. Les résultats de cette analyse HACCP sont détaillés dans les 2 annexes (annexe 1 : Liste des dangers potentiels, et annexe 2 : Analyse de risques).

\section{Champ d'application}

Le guide propose de bonnes pratiques $d$ 'hygiène aux opérateurs réalisant les opérations de :

- trituration des graines et fruits oléagineux (à l'exception de l'olive) ;

- raffinage des huiles brutes.

Ces bonnes pratiques d'hygiène s'étendent de la réception des graines et fruits oléagineux à l'expédition des produits finis, et contribuent à la sécurité et la salubrité des marchandises. Elles concernent l'ensemble des produits destinés à l'alimentation humaine et animale issus des activités de trituration de graines oléagineuses (colza, tournesol, soja, etc.) et des fruits oléagineux (noix, noisettes...), et des activités de raffinage. II s'agit notamment des tourteaux, des huiles brutes et raffinées, des coques, etc. Le transport des produits issus des activités trituration/raffinage n'est pas couvert par ce guide.

La mise en œuvre des recommandations de ce guide a pour objectif de réduire les risques de contamination biologique (salmonelles, etc.), chimique (résidus de produits phytosanitaires, métaux lourds...) et physique (corps étrangers : verre, pièce métallique...). Les risques que pourraient encourir des individus sujets aux allergies alimentaires sont pris en compte. En revanche, les exigences relevant du cadre commercial (OGM, etc.) n'ont pas été incluses dans ce guide. Ce guide, en particulier l'analyse de risques, ne couvre pas les huiles alimen- taires qui sont destinées, en tant qu'ingrédient, à l'industrie de l'alimentation infantile (baby food).

\section{Liste des dangers retenus}

Après étude des dangers biologiques, chimiques, physiques ou allergènes potentiels, notamment décrits dans les documents ANSES, Huileries de France a retenu une liste de dangers applicables pour les opérateurs de la trituration ou du raffinage (tableau 2).

Au vu du caractère anhydre de l'huile, un certain nombre de dangers biologiques (par exemple bactéries ou moisissures) ont été écartés. Seuls les tourteaux peuvent être exposés à de tels dangers, comme le développement de salmonelles. En revanche, les molécules lipophiles présentes à un stade antérieur à la trituration peuvent se concentrer dans I'huile brute (par exemple certains pesticides liposolubles), et constituent un paramètre important à surveiller. Parmi les dangers chimiques on peut distinguer :

- les contaminants issus de l'environnement, qui sont par exemple transmis du sol à la graine (mycotoxines, éléments traces métalliques, HAP...);

- les contaminants de process (produits de dératisation, produits détergents ou de nettoyage, composés aromatiques issus de l'hexane, etc.).

La présence d'entrepôts et d'usines a mené à retenir les dangers physiques tels que la présence de nuisibles type rongeurs ou d'insectes. II faut également vérifier pendant le processus de fabrication, quel qu'il soit, qu'aucun corps étranger ne soit demeuré dans les graines triturées.

Enfin un danger relatif aux allergènes a été retenu à cause du risque de contamination croisée de l'huile avec d'autres ingrédients allergènes utilisés dans l'usine (par exemple fruits à coque).

\section{Bonnes pratiques recommandées}

De bonnes pratiques générales $\mathrm{d}$ 'hygiène sont recommandées, avant même la mise en place du système HACCP. Elles concernent principalement des recommandations relatives:

- à I'hygiène des bâtiments et installations : se conformer à la réglementation aux installations classées si besoin ; l'implantation des bâtiments doit per- 
Tableau 2. Dangers potentiels retenus pour une étude HACCP pour les process de trituration et raffinage.

\begin{tabular}{|c|c|}
\hline Types de dangers & Dangers \\
\hline \multirow{4}{*}{ Biologiques } & Salmonelles \\
\hline & Alcaloïdes (Datura) \\
\hline & Moisissures \\
\hline & Impuretés botaniques \\
\hline \multirow{16}{*}{ Chimiques } & Résidus de produits phytopharmaceutiques \\
\hline & Mycotoxines \\
\hline & Eléments traces métalliques \\
\hline & $\overline{\mathrm{HAP}}$ \\
\hline & Dioxine/PCB \\
\hline & Radioactivité \\
\hline & Produits de dératisation \\
\hline & Détergents et produits de nettoyage \\
\hline & Phtalates \\
\hline & Composés aromatiques issus de l'hexane \\
\hline & Graisses/Huiles minérales de lubrification \\
\hline & Eau contaminée \\
\hline & Glucosinolates \\
\hline & Auxiliaires technologiques aux critères de pureté non conformes \\
\hline & Acide érucique \\
\hline & $\begin{array}{l}\text { Vapeur contaminée par des produits de traitement } \\
\text { des eaux de chaudière }\end{array}$ \\
\hline \multirow{3}{*}{ Physiques } & Corps étrangers \\
\hline & Insectes/acariens \\
\hline & Corps étrangers issus de nuisibles \\
\hline Allergènes & Ingrédients allergènes \\
\hline
\end{tabular}

mettre maintenance et nettoyage ; recommandation de séparer par fonctions et selon les flux de trituration/ raffinage; plan de dératisation important pour lutter contre les nuisibles ;

- à l'hygiène et au nettoyage des équipements de production : ils doivent être facilement nettoyables, avec un plan de maintenance; utilisation de matériaux ou substances aptes au contact alimentaire ; les produits de nettoyage/ désinfection doivent être conformes à la réglementation en vigueur ;

- à I'hygiène du personnel : formation du personnel à l'hygiène, importance des procédures en place ;

- aux intrants : les matières premières entrantes doivent être contrôlées (cahier des charges, respect de la réglementation, conditions de stockage,...) et un plan d'échantillonnage et d'analyse doit être mis en place ;

- au stockage et au chargement/ expédition des produits: éviter le mélange des produits lors du stockage
De nouvelles réglementations sont apparues au cours des deux dernières années (par exemple: HAP, ou dioxines et $P C B$ dont les teneurs maximales dans les denrées alimentaires ont été revues), nécessitant une mise à jour des procédures dans les entreprises.

Les dangers les plus délicats à gérer sont les contaminants émergents ne faisant pas l'objet d'une réglementation mais dont les effets délétères ont pu être mis en évidence. Les entreprises ont donc intégré dès que possible ces substances dans leur plan de surveillance. II est cependant difficile de déterminer une valeur maximale pour des substances n'ayant pas toujours fait l'objet d'une évaluation scientifique sur les doses journalières admissibles.

\section{Conclusion}

Le guide doit être un document de référence pour les opérateurs pour la maîtrise des risques sanitaires liés aux activités de I'huilerie. II constitue également un outil pratique pour le respect des réglementations en vigueur relatives à l'hygiène et à la maîtrise de la sécurité sanitaire des produits.

Il est en accord avec les exigences du guide EFISC, une certification européenne relative à la sécurité des ingrédients utilisés en alimentation animale développée par: I'Association des Amidonniers et Féculiers (AAF), la Fédération Européenne de I'Industrie des huiles et tourteaux (FEDIOL) et la Plateforme Européenne des principaux producteurs $d$ 'ingrédients destinés à I'alimentation animale (EFIP).

\section{Conflits d'intérêts : aucun} les dangers potentiels, pour déterminer s'ils doivent être considérés comme des dangers significatifs ou non dans I'industrie de I'huilerie. Comme pour le guide relatif à la collecte et au stockage (cf. chapitre précédent), le calcul prend en compte la fréquence et la gravité du danger considéré, avec une échelle allant de 1 à 4 .

Les programmes prérequis retenus pour les activités de trituration et raffinage concernent en priorité les salmonelles pour les tourteaux, la maintenance des machines pour éviter l'introduction de lubrifiants non alimentaires dans les produits, et le contrôle des matières premières vis-à-vis des contaminants (par exemple aflatoxines ou pesticides).

\section{RÉFÉRENCES}

Cerf O, Donnat E, the FarmHACCP Working Group. Application of hazard analysis Critical control point (HACCP) principles to primary production: what is feasible and desirable? Food Control 2011 ; 22 : 1839-43.

Règlement (CE) no 852/2004 du 29 avril 2004 relatif à l'hygiène de denrées alimentaires.

Règlement (CE) no 183/2005 du 12 janvier 2005 relatif à I'hygiène des aliments pour animaux.

Avis aux professionnels de l'alimentation relatif aux guides de bonnes pratiques d'hygiène et d'application des principes HACCP. $J O n^{\circ} 138$ du 15 juin 2005 page 10289 texte $n^{\circ} 127$. 